isted in the neighbourhood of the stricture rendered it impossible to get an instrument with the sharp curve of a sound into the bladder. Examination by the rectum discovered a prostatic tumour, about the size of a walnut, situated posterior and slightly to the left of the urethra,in fact, directly in the way of the lateral incision. From the hardness of the tumour, I thonght it might be a prostatic calculus. Examination of the urine showed it to be free from albumen and tube-casts; but pus and oxalate crystals existed in abundance. The patient also drew my attention to a very profuse purpuric rash which covered his legs, and $I$ found that his gums were spongy.

His sufferings were very great; he had not been able to work for many months; the stricture had existed for twelve years, and the fistula-which consisted of two tracks leading from behind the triangular ligament forward to the posterior edge of the scrotum-and purpura had existed many months. Altogether the conditions were nearly as unfavourable as it is well possible to imagine. Crushing was utterly out of the question, and I resolved to cut. But there was before my eyes the fear of hæmorrhage owing to the purpura. He therefore went back to the country, continued the appropriate treatment employed by Mr. Hirst, and returned in about a month, somewhat improved in health.

On the morning of March 2nd, when I had resolved to operate, he presented, however, a fresh erop of purpuric rash, a circumstance which very nearly prevented me from proceeding; but with the kind co-operation of Messrs. Hirst, Hollings, G. F. Naylor, and other friends, I accomplished the following operation:-

Having passed a small curved staff in to the bladder, I passed a probe down that fistulous track nearer the middle line, and, slitting up its posterior third, my knife entered the staff as if for perineal section, divided the stricture, was then turned as for the lateral incision, and passed on to the prostate. Here I found a little difficulty in keeping the knife in the groove of the staff; but with care I passed the knife into the bladder, cutting through an abnormally re. sisting structure. Following with my left forefinger, I at once recognised this structure as a very large instance of the prostatic myoma described by Thompson and Virchow, and by the removal of which, in certain cases, Sir William Fergusson has recently added something new to the operation of lithotomy, and not a little to his already immense reputation for skill and daring in operative surgery. My finger next recognised that, instead of two stones, the bladder was occupied by one large calculus of an oblong ovoid shape, and with a circular constriction in the middle, which had conveyed to me the false impression of the presence of two calculi. Had I been able to introduce a sound into the bladder, I might have avoided this mistake. The calculus proved to be a mulberry specimen, weighing five hundred grains. Introducing a probe-pointed bistoury along my finger, I extended the lateral incision throngh the tumour to an extent which I thought needful for the extraction of the stone, and the result was that I either completely or almost completely bisected the tumour, which was very resisting, and projected a good deal into the bladder. The removal of the stone was not difficult, but needed a little dragging. After its removal, examination of the wound showed me that the segments of the tumour had been partially dislodged - that it was capsulated, and might be removed. I was strongly inclined to attempt its removal; but, having waited a few minutes, and found that there was no bleeding, I hesitated to run further risk, and, remarking that the section of the tumour might lead to its atrophy, I desisted from further interference with it. I think that the result justified my discretion, because oozing of blood went on for three days, when it was checked by a few doses of turpentine. Had I torn across a small artery in the removal of the tumour in a position where I could not have reached it, the result might have been fatal. It is, however, as Sir William Fergusson has written to me on the subject, all well that ends well, and the patient went home on the seventeenth day with the wound all but healed, the fistulous tracks closed, and No. 9 catheter could be passed easily into bis bladder.

With reference to the tumour, Sir. William writes that he would probably have removed it, and he rould, of course, have been justified in so doing. But, for my part, this was a point which was decided mainly on seltish grounds. I did not dare to run the risk of any further complication-a risk which would have been nothing to the Professor of Surgery at King's College. Sir William has given me his opinion that the tumour will probably not atrophy, against my own hope that it might, as similar growths sometimes do in the uterus after an incision.

Wakefield, Mareh, 1870.

\section{ON A CASE OF MALIGNANT SCARLET FEVER.}

DEATH IN FORTY HOURS.

By J. J. THOMPSON, F.R.C.S.I., SURGEON 2ND BATT. 9TH REGIMENT.

THe interesting lecture on Scarlet Fever delivered by Sir Wm. Jenner at University College Hospital, and published in The LanceT of January 8th last, induces me to send for publication a case which occurred in this garrison lately, as it was very similar to some of the cases alluded to by that physician in his lecture.

The case which has come under my observation is remarkable, as showing how rapidly fatal the malignant form of scarlet fever may prove in a young and otherwise healthy man. Such was the intensity of the blood-poison in this instance that the man seemed suddenly struck down by it, and the case was evidently hopeless from the first. As has been so often noticed by other observers, the urgency of the local throat affection was by no means proportionate to the virulence of the constitutional phenomena.

On Thursday evening, Feb. 10th, 1870, at six o'clock, a young recruit from the depôt brigade of Foot Guards, in garrison at Warley, reported himself sick, and was at once admitted to hospital. He had been quartered at Warley since December last, and had the appearance as well as the character of being a strong and healthy man. He attended his usual drill the same afternoon (Thursday), and it was merely remarked by the drill corporal that he was not as smart as usual. There is no history of contagion, and the period of incubation must be conjectural.

On admission, he complained of his throat being very sore and of a general feeling of illness. When seen directly afterwards, he was lying on his back, low in the bed; his face was congested; eyes suffused; pulse small and frequent; and his tongue was covered with a dirty, creamy coat.

Next morning (Friday) the constitutional symptoms were of a marked asthenic character; pulse 120, feeble and quick; tongue dry and brown in centre; face of a dusky leaden hue, and the abdomen and chest were covered with a dusky red efflorescence; the fauces, uvula, and tonsils were livid and swollen. Towards the evening he became worse, and during the night delirium, not of a violent character, was incessant, and he died at ten o'clock on Saturday morning, about forty hours from the time of his admission.

Wine and beef-tea were freely given from the first, as well as chlorate of potash and diffusable stimulants.

Warley Barracks, Essex, Feb. 1870.

PRIMARY AMPUTATION OF SHOULDERJOINT SUCCESSFULLY PERFORMED AT THE PATIENT'S HOME; RLCOVERT.

\section{BY C. E. SAUNDERS, M.D.}

AT a time when the relative merits of hospital and home practice, as affecting capital operations, are under discussion, the following case of primary amputation may prove of some interest. Occurring, as it did, in a house the general arrangements of which were none too well regulated; nursed by zealous, it is true, but by no means skilled, hands; while it had, on the favourable side of the question, the all-important advantages of pure air, quiet, and, to a patient of such tender years, familiar surroundings, it offers, I think, a fair case for comparison.

On August 12th, 1869, I was summoned to attend $H$. 
$\mathrm{H}-$ a child of two and a half years old, who, about two hours previously, while playing in the road, had been knocked down, and his left arm run over by a waggon heavily laden with deals (the combined weight of which was between five and six tons). All other parts of his body escaped. He immediately jumped up, and ran across the road to his mother, who, engaged in conversation with a neighbour, had not witnessed the accident.

I found him sleeping quietly. The surface of the body was warm, and his pulse regular, and of fair power. There had been no great hæmorrhage, and it had now entirely ceased. On examining the arm, it was found that $\mathrm{jt}$ had sustained the following injuries. The skin and superficial fascia, from an inch below the bend of the elbow to the acromion process of the scapula, was cleanly dissected off the muscles beneath in the entire circumference of the limb. A wound, on the anterior aspect of this detached skin, extended from its commencement to the middle of the arm, and through this the humerus projected, fractured obliquely from above downwards, and within outwards; the upper extremity of fracture extending as high as the anatomical neck of the humerus, and slightly lacerating the capsular ligament. The lower fragment was separated from the upper by an interval of three inches, the continuity of the limb being alone maintained by a tubular portion of skin and superficial fascia; the muscles, arteries, and nerves having been completely torn across. From the character of these injuries, it would seem that the waggon must have rolled the arm round-and from the breadth of the wheel (four inches and a half), nearly the length of the arm itself, one can easily understand how this might happen,-the skin alone, from its elasticity, resisting the crushing weight, and only lacerated by the broken fragment of bone. On dissecting the limb after amputation, it was found that the condyles were fractured transversely, and the edge of the olecranon fossa crumbled down.

The little patient being placed under the influence of chloroform by Dr. Byass, I proceeded to remove the limb. Standing behind the patient, I entered my knife behind the spine of the scapula, at the posterior border of axilla, and carrying it across the anterior aspect of the joint, brought it out at the coracoid process, and made the anterior flap. This being raised by an assistant, the capsule of the joint was opened, and the muscles attached to the tuberosity were divided. Owing to the small fragment of bone remaining, little more than the head of the humerus, it was not easy to effect disarticulation. This being done, however, and keeping my knife close to the bone, I made the posterior flap, but waited to divide the axillary artery until kay assistant, Mr. Wright, cleverly following my knife closely up, had the artery completely under control. By these precautions, not more than half an ounce of blood was lost during the whole of the operation-a point which could not fail to have a favoureble influence on the success of the case. The anterior flap was composed of a double layer of skin dissected up at the time of the accident, as before described, and the mass of muscle, bruised and lacerated however, made by my incision. From this I anticipated some trouble, but was agreeably disappointed. The axi ilary artery alone required a ligature. Three sutures were put in, and the stump lightly bandaged.

On examining now the body for any other injury, it was noticed that the external meatus of the left ear was filled with blood; and, in connexion with the fact that the child was very drowsy, I was led to fear that some injury to the skull had occurred. I wiped the blood away, but again it appeared. I did not, however, make quite sure that the meatus beyond the range of casual observance was clear, and from the bleeding not recurring after my departure, and no head symptoms having manifested themselves, I conclude now that the blood had simply trickled in at the time of accident or operation. Feeling, however, a doubt at the time, I had the head shaved and applied ice.

Aug. 13th.-Has had some disturbed sleep; very feverish; pulse small and rapid; subsultus tendinum; tongue coated with white fur ; has passed urine.

14th.--Bowels moved; has been restless, but had a little sleep. To have saline mixture. Wound dressed; a quantity of dark serous fluid distending the flaps; edges of flaps look healthy.

15th.--Bruising behind shoulder-joint more apparent; pad placed over anterior flap to prevent collection of fluid, which still looks like dark-coloured serum. The value of well-arranged pads over a deeply seated, pus-forming cavity, preventing, as it does, collection of matter, and affording support for the new granulations, cannot be too highly esteemed, and is preferable, in $\mathrm{my}$ opinion, where practicable, to the drainage-tube, valuable as this is in sinuses. This rule of practice I have to thank my old master, $\mathrm{Mr}$. Solly, for inculcating. One suture was removed to give freer vent to discharge. Pulse becoming quieter and tongue cleaning.

16th.-Sleeps well ; fever abating; takes broth and milk freely; sits up and feeds himself; is cheerful, and notices his friends. Another suture removed. Warm-water dressing to be applied three times a day.

17th. - Discharge becoming thicker, and more like laudable pus. On gently raising the edge of the flap, granulations are seen clean and florid. A lotion of carbolic acid used when the arm is dressed.

18th.-Has a slight attack of bronchitis ; bowels daily relieved.

22nd-Cough better; sleeps well at night; seldom complains of pain in the arm.

24th.-Wound in middle half healed; not much discharge, and that healthy.

28th.-Ligature came away.

Sept. 2nd.-Wound healing, but great tendency to collection of matter; the pads being still indispensable.

10th.-Wound to be syringed with tincture of benzoin and rectified spirits of wine, equal parts.

17th.-Wound nearly healed in whole extent. The patient is up and dressed.

30th.--Runs about quite convalescent, and wound soundly healed.

Cuckfield, Sussex, Mareh, 1870.

ON THE USE OF

NITROUS OXIDE GAS AS AN AN ASTHETIC IN SURGERY, WITH COXETER'S LIQUID GAS.

BY CHAS. JAS. FOX, M.R.C.S., L.D.S., DENTAL SURGEON TO THE DENTAL HOSPITAL OF LONDON AND TO THE GREAT NORTHERN HOSPITAL.

Ir may be fairly assumed that nitrous oxide is now admitted by all who have had any experience with it whatever to be a safe, rapid, and effectual anæsthetic for short operations. I use the term anæsthetic as it is generally understood, without entering upon the question as to whether the gas is or is not a true anæsthetic. That it possesses the power of inducing a condition in which painful operations may be effected without the patient's cognisance is an undeniable fact, and sufficient for my present purpose.

It is difficult to witness daily the delight of those who, in my specialty, have experienced the relief afforded by the gas, and compare it with the torpid misery of those who have been relieved from their pain under the influence of chloroform, without feeling an almost irrepressible desire to see the comfort of the gas extended to those who have to undergo more severe operations. This must be my excuse for entering upon a field which might be more fitly occupied by some of those whose practice is more exclusively devoted to general surgery; but I am so impressed with the belief that this anæsthetic would, if properly and fairly tried in general surgery, prove an inestimable boon in thousands of cases, that I cannot refrain from endeavouring to turn the attention of medical men more earnestly to the subject. I am far from desiring to depreciate the value of chloroform; in many cases it will continue to be the only anæsthetic that can or may be used; and, if I may be allowed a short digression, $I$ would add that the rapidity with which a patient is anæsthetised by it is as much rreater, and his recovery therefrom is as much more satisfactory, when Mr. Clover's apparatus is employed instead of any other mode of inhalation, as-so far as my experience goes-the effect of nitrous oxide is more satisfactory on both points, than that of chloroform by any mode of administration. 